\title{
Rewiring cellular networks
}

RNA-based regulatory systems control the behavior of cells in response to endogenous proteins.

Cells are built up of complex molecular networks that control the cell's responses to the environment and cell-fate decisions such as proliferation, differentiation or apoptosis. These molecular networks process information and link it to new cellular behaviors. To study and manipulate the wiring that underlies cellular function, one needs tools that allow interfering with these signaling networks in a precise and controlled manner.

Several different bioengineering strategies now allow researchers to do this. These perturbative tools are normally composed of a 'sensing' module that responds to particular stimuli and an 'effector' module that alters cellular behavior by affecting processes such as gene expression, enzymatic activity or protein translocation.

Christina Smolke and her colleagues at Stanford University are interested in using RNA for building such tools. In new work, her group has engineered 'RNA controllers', molecules that respond to specific cellular proteins by modulating the expression of other proteins. "We took aptamers-which are basically RNA molecules that will bind to specific proteins - and used them as our sensing components and then linked them to an RNA-based gene regulatory system based on alternative splicing," she explains.

In these RNA devices, a gene of interest is placed downstream of a minigene composed of three coding regions (exons) separated by two noncoding regions (introns) that contain the aptamer. The middle exon has a stop codon such that translation of the gene of interest is high when the exon is excluded. The binding of a protein to the aptamer affects the splicing pattern of this RNA molecule through mechanisms that are to be determined, resulting in changes in expression of the gene of interest. The system is highly flexible: depending on where the aptamer sequence is placed in the intron, its binding to the protein can affect splicing differently, increasing or decreasing the final levels of the gene product. "You can also swap in different sensors [aptamers] that respond to different proteins in the cell and maintain the same sort of functional genetic device," says Smolke, or build devices that respond to multiple protein inputs by incorporating several aptamers, as is shown in this work.

RNA controllers that respond to both endogenous and heterologous nuclear proteins can be built in this way. Graduate student Stephanie Culler and postdoc Kevin Hoff in Smolke's laboratory engineered RNA devices that respond to proteins involved in the NF- $\kappa B$ and Wnt signaling pathways showing, in human cultured cells, that they could rewire these pathways and produce new cellular behaviors. By placing a suicide gene as the effector module of $\beta$-catenin and $\mathrm{NF}-\kappa \mathrm{B}$ responsive devices, the group could trigger targeted cell death upon stimulation of these pathways.

Smolke is interested in integrating RNA controllers in different biological systems. By looking at how these devices can be used to control signaling pathways that affect the cell cycle, for example, her group is looking ahead to their future application for disease treatment and stem-cell engineering.

\section{BIOCHEMISTRY}

\section{BETTER LIVING THROUGH BIOCHEMISTRY}

In-depth mass spectrometric analysis reveals how cells survive stress by coordinating various enzymes that modify RNAs involved in protein synthesis.

Despite the remarkable functional diversity that can be achieved with just four bases, many RNA molecules also undergo diverse targeted chemical modifications that considerably expand the nucleotide dictionary. Transfer RNA (tRNA) molecules are particularly prone to alteration, with nearly 100 base variants identified in tRNAs from a broad range of species.

Several of these changes have been individually associated with tRNA stability or translational efficiency, but a new study now reveals how large-scale coordination of RNA modification may enable cells to modulate the production of subsets of proteins in response to specific external stimuli (Chan et al., 2010).

This project was launched several years ago, after Thomas Begley's team at the State University of New York at Albany determined that for yeast cells to survive exposure to the toxic alkylating agent methane methylsulfonate (MMS) they require tRNA methyltransferase 9 (Trm9), an enzyme that modifies uracil nucleotides in the anticodon of specific glutamate and arginine tRNAs (Begley et al., 2007). Notably, the codons recognized by these tRNAs were especially abundant in gene sequences that participate in the cellular response to MMS, suggesting that this may be a specialized protective mechanism against toxic stress.
Begley then partnered with Massachusetts Institute of Technology bioanalytical chemist Peter Dedon and graduate student Clement Chan to perform a far broader analysis of the dynamic regulation of 23 different tRNA modifications known to occur in Saccharomyces cerevisiae. "We decided to look at this systematically and see if there are patterns here that relate to the cellular response to virtually any stimulus," says Dedon.

They developed a rigorous, high-sensitivity approach pairing liquid chromatography and mass spectrometry that enabled them to characterize and quantify all of these various yeast tRNA modifications in parallel-including rare alterations that have proven difficult to quantify in the past-in response to treatment with varying concentrations of MMS and three other toxic compounds. The resulting data revealed clear and highly reproducible chemical- and dose-specific changes in the levels of specific subsets of nucleotide variants. In parallel, Begley and Dedon demonstrated that the targeted deletion of genes encoding individual tRNA-modifying enzymes could markedly increase the specific sensitivity of yeast cells to each toxin, indicating that the protective response previously identified for Trm9 represents a general functional role for such modifications. "Our study shows the dynamics of these modifications, and how groups of these modifications are coordinated during cellular stress," says Begley. By correlating these data with their mass spectrometry results, the 


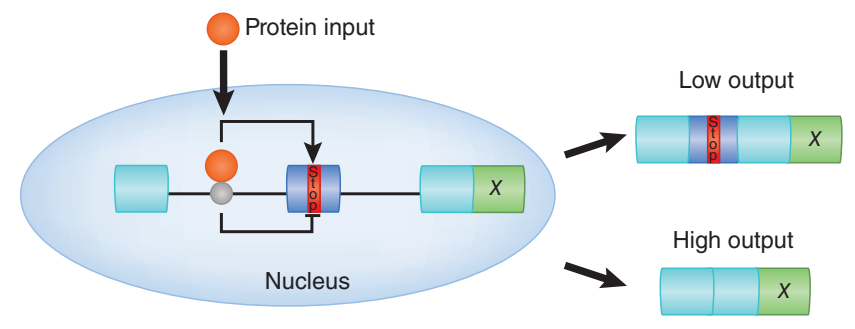

Mechanism of action of RNA controllers. Binding of the protein input to the sensor alters the splicing pattern of the RNA molecule by enhancing or suppressing alternative exon exclusion, resulting in different production levels of the gene of interest, $X$.

These RNA-based devices are not only one of the first ones demonstrated to respond to native proteins but also, because they are based on the use of alternative splicing, they offer endless possibilities for gene-expression regulation. One could imagine future developments of more complex types of controllers based on similar strategies as the one used by the Smolke group. For example, by regulating the inclusion of exons that actually encode for functional protein domains, one could control what splicing variant of a protein is produced or where it is localized in the cell. Bioengineering and synthetic biology are rapidly growing fields and their application to biological studies will enable fine control of cellular function and also aid in further refining these promising techniques.

\section{Erika Pastrana}

\section{RESEARCH PAPERS}

Culller S. J. et al. Reprogramming cellular behavior with RNA controllers responsive to endogenous proteins. Science 330, 1251-1255 (2010).

researchers could even piece together new biosynthetic pathways underlying the production of several nucleotide variants.

However, they also encountered unexpected complexity, with many apparent disparities between the biochemical and genetic data. For example, even though levels of nucleotide variant $\mathrm{M}^{2}{ }_{2} \mathrm{G}$ increased in response to hydrogen peroxide, cells lacking tRNA methyltransferase 1 , the enzyme responsible for this particular modification, were not notably sensitized to hydrogen peroxide treatment. Dedon points out that clarifying the mechanistic explanations for these seeming contradictions and delving deeper into how these pathways specifically intersect and interact to achieve specific biological outcomes will be primary objectives for future work. The resulting data will ultimately be incorporated into a publicly accessible repository.

In parallel, they are also moving outside of eukaryotic organisms to explore the extent to which pathogenic bacteria may exploit such modification pathways to flourish in their hosts. "When a macrophage produces reactive nitrogen species, how do bacteria respond to these toxic chemicals in terms of translational control mechanisms?" asks Dedon. "We're very eager to look at the microbial side of this whole RNA modification story."

\section{Michael Eisenstein}

\section{RESEARCH PAPERS}

Begley, U. et al. Trm9-catalyzed tRNA modifications link translation to the DNA damage response. Mol. Cell 28, 860-870 (2007).

Chan, C.T.Y. et al. A quantitative systems approach reveals dynamic control of tRNA modifications during cellular stress. PLoS Genet. 6, e1001247 (2010).

\section{BIOCHEMISTRY}

\section{An RNA crystallization chaperone}

The crystallization of RNA molecules for structural analysis is even more challenging than protein crystallization owing to the low chemical diversity, flexibility and conformational heterogeneity of RNA. Koldobskaya et al. introduce a chaperone system that stabilizes RNA structure and promotes crystallization. The chaperone is an antigen-binding fragment (Fab) that recognizes an epitope tag that can be installed on any RNA of interest. Koldobskaya, Y. et al. Nat. Struct. Mol. Biol. 18, 100-106 (2011).

\section{BIOPHYSICS}

\section{Video force microscopy}

Brodland et al. introduce a technique called video force microscopy, which is used to noninvasively generate detailed force maps of tissues from time-lapse multiphoton images. The method involves discretizing tissue into polygonal regions and tracking the corners of these regions to quantify deformations, allowing the user to determine whether the deformations are a result of active or passive forces. Brodland et al. used the method to measure the driving forces responsible for ventral furrow formation in Drosophila melanogaster embryos.

Brodland, G.W. et al. Proc. Natl. Acad. Sci. USA 107, 22111-22116 (2010).

\section{EPIGENETICS}

\section{Distribution of 5-hydroxymethylcytosine in the genome}

As 5-hydroxymethylcytosine (5-hmC) is a recently discovered epigenetic modification, little is currently known about its distribution and functional roles. Song et al. have now developed a method for mapping the genome-wide distribution of 5-hmC. The method involves using a $\beta$-glucosyltransferase to transfer a glucose containing an azide handle onto 5 -hmC; the azide can then be tagged with biotin for enrichment and sequencing of the captured DNA fragments containing 5-hmC.

Song, C.-X. et al. Nat. Biotechnol. 29, 68-72 (2011).

\section{PROTEOMICS}

\section{Protein-lipid interactions}

Besides building cellular membranes, lipids operate as important signaling molecules via their interactions with proteins, but knowledge of these interactions is incomplete. Gallego et al. report a systematic, unbiased screening approach to identify protein-lipid interactions in the yeast Saccharomyces cerevisiae by detecting protein binding to miniature lipid arrays. Gallego et al. identified 530 protein-lipid interactions, most of which were previously unknown.

Gallego, 0. et al. Mol. Syst. Biol. advance online publication, 30 November 2010.

\section{CELL BIOLOGY}

\section{Carbon nanotube endoscopes}

Glass pipettes are useful tools for making electrophysiological measurements and injecting substances into cells, but because of their large size they can damage the cell. Singhal et al. introduce a carbon nanotube-based 'endoscope', 10 times smaller than a typical glass pipette, which can be used to probe cells with 100-nanometer spatial resolution, including intracellular organelles. The endoscope can also be used to deliver fluids to the cell, something previous carbon nanotubebased probes could not do.

Singhal, R. et al. Nat. Nanotechnol. 6, 57-54 (2011). 